\title{
XXVII. A comparison of the electric theory of light and Sir William Thomson's theory of a quasi-labile Ether
}

\section{J. Willard Gibbs}

To cite this article: J. Willard Gibbs (1889) XXVII. A comparison of the electric theory of light and Sir William Thomson's theory of a quasi-labile Æther, Philosophical Magazine Series 5, 27:166, 238-253, DOI: $10.1080 / 14786448908628344$

To link to this article: http://dx.doi.org/10.1080/14786448908628344

曲 Published online: 29 Apr 2009.

Submit your article to this journal $\pi$

Џll Article views: 5

Q View related articles $\llbracket$ 


\section{[ 238 ]}

XXVII. A Comparison of the Electric Theory of Light and Sir William Thomson's Theory of a Quasi-labile Ether. By J. Willard Gibbs*.

A REMARKABLE paper by Sir William Thomson, in has opened a new vista in the possibilities of the theory of an elastic æther. Since the general theory of elasticity gives three waves characterized by different directions of displacement for a single wave-plane, while the phenomena of optics show but two, the first point in accommodating any theory to observation is to get rid (absolutely or sensibly) of the third wave. For this end it has been common to make the æther incompressible, or, as it is sometimes expressed, to make the velocity of the third wave infinite. The velocity of the wave of compression becomes, in fact, infinite as the compressibility vanishes. Of course it has not escaped the notice of physicists that we may also get rid of the third wave by making its velocity zero, as may be done by giving certain values to the constants which express the elastic properties of the medium ; but such values have appeared impossible, as involving an unstable state of the medium. The condition of incompressibility, absolute or approximate, has therefore appeared necessaryt. This question of instability has now, however, been subjected to a more searching examination, with the result that the instability does not really exist " provided we either suppose the medium to extend all through boundless space, or give it a fixed containing-vessel as its boundary." This renders possible a very simple theory of light, which has been shown to give Fresnel's laws for the intensities of reflected and refracted light and for double refraction, so far as concerns the phenomena which can be directly observed. The displacement in an æolotropic medium is in the same plane passing through the wave-normal, as was supposed by Fresnel; but its position in that plane is different, being perpendicular to the ray instead of to the wave-normal .

* From the American Journal of Science for February 1889.

$\dagger$ It was under this impression that the paper entitled "A Comparison of the Elastic and the Electric Theories of Light with respect to the Law of Double Refraction and the Dispersion of Colours," in the June number of the Amer. Journ. Sci., was written. The conclusions of that paper, except so far as respects the dispersion of colours, will not apply to the new theory.

† Sir William Thomson, loc. cit. R. T. Glazebrook, Phil. Mag. December 1888 . 
It is the object of this paper to compare this new theory with the electric theory of light. In the limiting cases (that is, when we regard the velocity of the missing wave in the elastic theory as zero, and in the electric theory as infinite) we shall find a remarkable correspondence between the two theories; the motions of monochromatic light within isotropic or æolotropic media of any degree of transparency or opacity, and at the boundary between two such media, being represented by equations absolutely identical, except that the symbols which denote displacement in one theory denote force in the other, and vice vers $\hat{a}$ *. In order to exhibit this correspondence completely and clearly, it is necessary. that the fundamental principles of the two theories should be treated with the same generality, and, so far as possible, by the same method. The immediate consequences of the new theory will therefore be deduced with the same generality and essentially by the same method which has been used with reference to the electric theory in a former volume of the American Journal of Science (vol. xxv. p. 107).

The elastic properties of the æther, according to the new theory, in its limiting case, may be very simply expressed by means of a vector operator, for which we shall use Maxwell's designation. The curl of a vector is defined to be another vector so derived from the first that if $u, v, w$ be the rectangular components of the first, and $u^{\prime}, v^{\prime}, w^{\prime}$ those of its curl,

$$
u^{\prime}=\frac{d w}{d y}-\frac{d v}{d z}, \quad v^{\prime}=\frac{d u}{d z}-\frac{d w}{d x}, \quad w^{\prime}=\frac{d v}{d x}-\frac{d u}{d y}, .
$$

where $x, y, z$ are rectangular coordinates. With this understanding, if the displacement of the æther is represented by the vector $(E$, the force exerted upon any element by the surrounding æther will be

$$
\text { -B curl curl E } d x d y d z, \text {. . . . }
$$

where $\mathrm{B}$ is a scalar (the so-called rigidity of the æther) having the same constant value throughout all space, whether ponderable matter is present or not.

Where there is no ponderable matter; this force must be equated to the reaction of the inertia of the xther. This gives, with omission of the common factor $d x d y d z$,

$$
\mathrm{A} \ddot{\mathfrak{\xi}}=-\mathrm{B} \text { curl curl } \vec{\xi}, \ldots . . .
$$

where A denotes the density of the æther.

* In giving us a new interpretation of the equations of the electric theory, the author of the new theory has in fact enriched the mathematical theory of physics with something which may be compared to the celebrated principle of duality in geometry. 


\section{Prof. J. W. Gibbs's Comparison of the Electric Theory}

The presence of ponderable matter disturbs the motions of the rether, and renders them too complicated for us to follow in detail. Nor is this necessary; for the quantities which occur in the equations of optics represent average values, taken over spaces large enough to smooth out the irregularities due to the ponderable particles, although very small as measured by a wave-length*. Now the general principles of harmonic motion $\dagger$ show that, to maintain in any element of volume the motion represented by

$$
\mathfrak{F}=\mathfrak{A} e^{2 \pi t} \frac{t}{p}, \ldots . . . \quad . \quad .
$$

$\mathfrak{A l}$ being a complex vector-constant, will require a force from outside represented by a complex linear vector-function of $\mathbb{F}$; that is, the three components of the force will be complex linear functions of the three components of $(\xi$. We shall represent this force by

$$
\mathrm{B} \Psi \ddot{\xi} d x d y d z, \text {. . . . . . }
$$

where $\Psi$ represents a complex linear vector-function $\neq$.

If we now equate the force required to maintain the motion in any element to that exerted upon the element by the surrounding æther, we have the equation

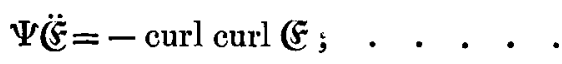

which expresses the general law for the motion of monochromatic light within any sensibly homogeneous medium, and may be regarded as implicitly including the conditions relating to the boundary of two such media, which are necessary for determining the intensities of reflected and refracted light.

* This is in no respect different from what is always tacitly understood in the theory of sound, where the displacements, velocities, and densities considered are always such average values. But in the theory of light it is desirable to have the fact clearly in mind, on account of the two interpenetrating media (imponderable and ponderable), the laws of light not being in all respects the same as they would be for a single homogeneous medium.

† See Lord Rayleigh's 'Theory of Sound,' vol. i. chapters iv., v.

I It amounts essentially to the same thing, whether we regard the force as a linear vector-function of $\mathbb{E}$ or of $\ddot{\xi}$, since these differ only by the constant factor $-\frac{4 \pi^{2}}{p^{2}}$. But there are some advantages in expressing the force as a function of $\ddot{\vec{E}}$, because the greater part of the force, in the most important cases, is required to overcome the inertia of the wether, and is thus more immediately connected with 
of Light and the Theory of a Quasi-labile Aither.

For let $u, v, w$ be the components of $(E$,

so that

$$
\begin{array}{lllll}
v^{\prime}, v^{\prime}, w^{\prime} & \# & \# & \# & \operatorname{curl}(\mathfrak{F}, \\
u^{\prime \prime}, v^{\prime \prime}, w^{\prime \prime} & \# & , & \# & \operatorname{curl} \operatorname{curl}(\mathfrak{F},
\end{array}
$$

$$
\begin{array}{ll}
u^{\prime}=\frac{d w}{d y}-\frac{d v}{d z}, & v^{\prime}=\frac{d u}{d z}-\frac{d w}{d x}, \quad w^{\prime}=\frac{d v}{d x}-\frac{d u}{d y} \\
u^{\prime \prime}-\frac{d w^{\prime}}{d y}-\frac{d v^{\prime}}{d z}, & v^{\prime \prime}=\frac{d u^{\prime}}{d z}-\frac{d w^{\prime}}{d x}, \quad w^{\prime \prime}=\frac{d v^{\prime}}{d x}-\frac{d u^{\prime}}{d y}
\end{array}
$$

and let the interface be perpendicular to the axis of $\mathrm{Z}$. It is evident that if $u^{\prime}$ or $v^{\prime}$ is discontinuous at the interface, the value of $u^{\prime \prime}$ or $v^{\prime \prime}$ becomes in a sense infinite, $i$. e. curl curl $(\mathbb{E}$, and therefore, by (6), $\Psi \ddot{\xi}$ will be infinite. Now both $\mathcal{E}$ and $\Psi$ are discontinuous at the interface, but infinite values for $\Psi \ddot{\vec{\xi}}$ are not admissible. Therefore $u^{\prime}$ and $v^{\prime}$ are continuous. Again, if $u$ or $v$ is discontinuous, $u^{\prime}$ or $v^{\prime}$ will become infinite, and therefore $u^{\prime \prime}$ or $v^{\prime \prime}$. Therefore $u$ and $v$ are continuous. These conditions may be expressed in the most general manner by saying that the components of $F$ and curl $(F$ parallel to the interface are continuous. This gives four complex scalar conditions, or in all eight scalar conditions, for the motion at the interface, which are sufficient to determine the amplitude and phase of the two reflected and the two refracted rays in the most general case. It is easy, however, to deduce from these four complex conditions two others, which are interesting and sometimes convenient. It is evident from the definitions of $w^{\prime}$ and $w^{\prime \prime}$ that, if $u, v, u^{\prime}$, and $v^{\prime}$ are continuous at the interface, $w^{\prime}$ and $w^{\prime \prime}$ will also be continuous. Now $-w^{\prime \prime}$ is equal to the component of $\Psi \ddot{\xi}$ normal to the interface. The following quantities are therefore continuous at the interface:-

the components parallel to the interface of $\mathbb{E}$, the component normal to the interface of $\Psi \mathbb{E}$, all components of . . . . . . . curl (E.)

To compare these results with those derived from the electrical theory, we may take the general equation of monochromatic light on the electrical hypothesis from a paper in a former volume of Silliman's American Journal. This equation, which with an unessential difference of notation may be written*

$$
\text { - Pot } \ddot{\mathfrak{F}}-\nabla \mathrm{Q}=4 \pi \Phi \mathscr{F}, . . \quad . \quad \text {. }
$$

was established by a method and considerations similar to those

* See the Amer. Journ. Sci. vol. xxv. p. 114, equation (12).

Phil. Mag. S. 5. Vol. 27. No. 166. March 1889. R 


\section{Prof. J. W. Gibbs's Comparison of the Electric Theory}

which have been used to establish equation (6), except that the ordinary law of electrodvnamic induction had the place of the new law of elasticity. $\mathscr{F}$ is a complex vector representing the electrical displacement as a harmonic function of the time; $\Phi$ is a complex linear vector-operator, such that $4 \pi \Phi \mathscr{F}$ represents the electromotive force necessary to keep up the vibration $\mathfrak{F} . \quad Q$ is a complex scalar representing the electrostatic potential ; $\nabla \mathrm{Q}$ the vector of which the three components are

$$
\frac{d \mathrm{Q}}{d x}, \frac{d \mathrm{Q}}{d y}, \frac{d \mathrm{Q}}{d z} .
$$

Pot denotes the operation by which, in the theory of gravitation, the potential is calculated from the density of matter*. When it is applied, as here, to a vector, the three components of the result are to be calculated separately from the three components of the operand. $-\nabla Q$ is therefore the electrostatic force, and - Pot $\ddot{\widetilde{F}}$ the electrodynamic force. In establishing the equation, it was not assumed that the electrical motions are solenoidal, or such as to satisfy the so-called "equation of continuity." We may now, however, make this assumption, since it is the extreme case of the electric theory which we are to compare with the extreme case of the elastic.

It results from the definitions of $\operatorname{curl}$ and $\nabla$ that curl $\nabla \mathrm{Q}=0$. We may therefore eliminate $Q$ from equation (8) by taking the curl. This gi res

$$
-\operatorname{curl} \text { Pot } \ddot{\mathscr{F}}=4 \pi \operatorname{curl} \Phi \mathfrak{F} . \quad . \quad . \quad . \quad \text {. }
$$

Since curl curl and $\frac{1}{4 \pi}$ Pot are inverse operators for solenoidal vectors, we may get rid of the symbol Pot by taking the curl again. We thus get

$$
-\ddot{\mathscr{r}}=\operatorname{curl} \operatorname{curl} \Phi \mathfrak{F} \text {. }
$$

The considerations for the motion at the houndary between different media are easily obtained from the following considerations. Pot $\ddot{\mathscr{F}}$ and $Q$ are evidently continuous at the interface. Therefore the components parallel to the interface of $\nabla Q$, and, by (8), of $\Phi \mathfrak{F}$, will be continuous. Again, curl Pot $\ddot{\mathfrak{F}}$ is continuous at the interface, as appears from the consideration that curl Pot $\dot{\mathscr{F}}$ is the magnetic force due to the electrical motions $\dot{\mathfrak{V}}$. Therefore, by $(9)$, curl $\Phi \mathscr{F}$ is con-

* The symbol - Pot is therefore equivalent to $4 \pi \nabla^{-2}$, as used by Sir William Thomson (with a happy economy of symbols) at the last meeting of the British Association to express the same law of electrodynamic induction, except that the symbol is here used as a vector operator. See 'Nature,' vol. xxxviii. p. 571, sub. init. 
tinuous. The solenoidal condition requires that the component of $\mathfrak{F}$ normal to the interface shall be continuous.

The following quantities are therefore continuous at the interface :-

the components parallel to the interface of $\Phi \mathscr{F}$ the component normal to the interface of $\mathscr{F}$, all components of . . . . . . . . curl $\Phi \mathfrak{F}$.

Of these conditions, the two relating to the normal components of $\mathfrak{F}$ and curl $\Phi \mathscr{F}$ are easily shown to result from the other four conditions, as in the analogous case in the elastic theory.

If we now compare in the two theories the differential equations of the motion of monochromatic light, for the interior of a sensibly homogeneous medium, (6) and (10), and the special conditions for the boundary between two such media as represented by the continuity of the quantities (7) and (11), we find that these equations and conditions become identical, if

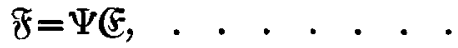

$$
\begin{aligned}
& \mathfrak{F}=\Phi \mathfrak{F}, \quad . \quad . \quad . \quad . \cdot \\
& \Psi=\Phi^{-1} \text {. . . . . . . . }
\end{aligned}
$$

In other words, the displacements in either theory are subject to the same general and surface conditions as the forces required to maintain the vibrations in an element of volume in the other theory.

To fix our ideas in regard to the signification of $\Psi$ and $\Phi$, we may consider the case of isotropic media, in which these operators reduce to ordinary algebraic quantities, simple or complex. Now the curl of any vector necessarily satisfies the solenoidal condition (the so-called " equation of continuity"), therefore by (6) $\Psi \mathfrak{F}$ and $(\mathcal{F}$ will be solenoidal. So also will $\mathfrak{F}$ and $\Phi \mathscr{F}$ in the electrical theory. Now for solenoidal vectors,

$$
-\operatorname{curl} \operatorname{curl}=\frac{d^{2}}{d x^{2}}+\frac{d^{2}}{d y^{2}}+\frac{d^{2}}{d z^{2}} ; \quad \therefore \quad .
$$

so that the equations (6) and (10) reduce to

$$
\begin{aligned}
\Psi \ddot{\mathfrak{F}} & =\left(\frac{d^{2}}{d x^{2}}+\frac{d^{2}}{d y^{2}}+\frac{d^{2}}{d z^{2}}\right) \mathfrak{F}, . . . \\
\ddot{\mathfrak{F}} & =\left(\frac{d^{2}}{d x^{2}}+\frac{d^{2}}{d y^{2}}+\frac{d^{2}}{d z^{2}}\right) \Phi \mathfrak{F} . \quad . \quad . .
\end{aligned}
$$

R 2 
For a simple train of waves, the displacement, in either theory, may be represented by a constant multiplied by

$$
e^{\iota(g t+a x+b y+c z)} \text {. . . . . . . }
$$

Our equations then reduce again to

Hence

$$
\begin{aligned}
g^{2} \Psi \mathfrak{F} & =\left(a^{2}+b^{2}+c^{2}\right) \mathfrak{F}, \quad . \quad . \quad . \quad . \quad . \\
g^{2} \mathfrak{Z} & =\left(a^{2}+b^{2}+c^{2}\right) \Phi \mathfrak{F} . \quad
\end{aligned}
$$

$$
\Psi^{-1}=\Phi=\frac{g^{2}}{a^{2}+b^{2}+c^{2}} . . \quad . \quad . \quad .
$$

'The last member of this equation, when real, evidently expresses the square of the velocity of light. If we set

$$
n^{2}=k^{2} \frac{a^{2}+b^{2}+c^{2}}{g^{2}}, \text {. . . . . }
$$

$k$ denoting the velocity of light in vacuo, we have

$$
n^{2}=k^{2} \Psi=k^{2} \Phi^{-1} \text {. . . . . . }
$$

When $n^{2}$ is positive, which is the case of perfectly transparent bodies, the positive root of $n^{2}$ is called the index of refraction of the medium. In the most general case it would be appropriate to call $n$ (or perhaps that root of $n^{2}$ of which the real part is positive) the (complex) index of refraction, although the terminology is hardly settled in this respect. A negative value of $n^{2}$ would represent a body from which light would be totally reflected at all angles of incidence. No such cases have been observed. Values of $n^{2}$ in which the coefficient of $\iota$ is negative, indicate media in which light is absorbed. Values in which the coefficient of $\iota$ is positive would represent media in which the opposite phenomenon took place*.

It is no part of the object of this paper to go into the details by which we may derive, so far as observable phenomena are concerned, Fresnel's law of double refraction for transparent bodies, as well as the more general law of the same character which relates to æolotropic bodies of more or less opacity, and which differs from Fresnel's only in that certain quantities become complex, or Fresnel's laws for the intensities of reflected and refracted light at the boundary of transparent isotropic media with the more general laws for the case of bodies æolotropic or opaque, or both. The principal cases have already been discussed on the new elastic theory

* But $\iota$ might have been introduced into the equations in such a way that a positive coefficient in the value of $n^{2}$ would indicate absorption, and a negative coefficient the impossible case. 
in the Philosophical Magazine*, and a further discussion is promised. For the electrical theory, the case of double refraction in perfectly transparent media has been discussed quite in detail in the Amer. Journ. Sci. $\dagger$; and the intensities of reflected and refracted light have been abundantly deduced from the above conditions by various authors $\neq$. So far as all these laws are concerned, the object of this paper will be attained if it has been made clear that the two theories, in their extreme cases, give identical results. The greater or less degree of elegance, or completeness, or perspicuity, with which these laws may be developed by different authors should weigh nothing in favour of either theory.

The nonmagnetic rotation of the plane of polarization, with the allied phenomena in æolotropic bodies, lie in a certain sense outside of the above laws, as depending on minute quantities which have been neglected in this discussion. The manner in which these minute quantities affect the equations of motion on the electrical theory has been shown in a former paper $\S$, where these phenomena in transparent bodies are treated quite at length. For the new theory, a discussion of this subject is promised by $\mathrm{Mr}$. Glazebrook.

But the magnetic rotation of the plane of polarization, with the allied phenomena when an æolotropic body is subjected to magnetic influence, fall entirely within the scope of the above equations and surface-conditions. The characteristic of this case is that $\Psi$ and $\Phi$ are not self-conjugatell. This is what we might expect on the electric theory from the experiments of Dr. Hall, which show that the operators expressing the relation between the electromotive force and current are not in general self-conjugate in this case.

In the preceding comparison, we have considered only the limiting cases of the two theories. With respect to the sense in which the limiting case is admissible, the two theories do not stand on quite the same footing. In the electric theory, or in any in which the velocity of the missing wave is very great, if we are satisfied that the compressibility is so small as to produce no appreciable results, we may set it equal to zero

* Sir William Thomson, loc. cit. R. T. Glazebrook, loc. cit.

t Vol. xxiiii. p. 262.

† Lorentz, Schlömilch's Zeitschrift, vol. xxii. pp. 1-30 and 205-219; vol. xxiii. pp. 197-210; Fitzgerald, Phil. Trans. vol. clxxi. p. 691 ; J. J. Thomson, Phil. Mag. [5] vol. ix. p. 284; Rayleigh, Phil. Mag. [5] vol. xii. p. 81. Glazebrook, Proc. Camb. Phil. Soc. vol. iv. p. 155.

\$ Amer. Journ. Sci. vol. xxiii. p. 460.

॥ See Amer. Journ. Sci. vol. xxv. p. 113. 


\section{Prof. J. W. Gibbs's Comparison of the Electric Theory}

in our mathematical theory, even if we do not regard this as expressing the actual facts with absolute accuracy. But the case is not so simple with an elastic theory in which the forces resisting certain kinds of motion vanish, so far, at least, as they are proportional to the strains. The first requisite for any sort of optical theory is that the forces shall be proportional to the displacements. This is easily obtained in general by supposing the displacements very small. But if the resistance to one kind of distortion vanishes, there will be a tendency for this kind of distortion to appear in some places in an exaggerated form, and even to an infinite degree, however small the displacements may be in other parts of the field. In the case before us, if we suppose the velocity of the missing wave to be absolutely zero, there will be infinite condensations and rarefactions at a surface where ordinary waves are reflected; that is, a certain volume of æther will be condensed to a surface, and vice versâ. This prevents any treatment of the extreme case, which is at once simple and satisfactory. The difficulty bas been noticed by Sir William Thomson, who observes that it may be avoided if we suppose the displacements infinitely small in comparison with the wave-length of the wave of compression. This implies a finite velocity for that wave. A similar difficulty would probably be found to exist (in the extreme case) with regard to the deformation of the æether by the molecules of ponderable matter, as the æther oscillates among them. If the statical resistance to irrotational motions is zero, it is not at all evident that the statical forces evoked by the disturbance caused by the molecules would be proportional to the motions. But this difficulty would be obviated by the same hypothesis as the first.

These circumstances render the elastic theory somewhat less convenient as a working hypothesis than the electric. They do not necessarily involve any complication of the equations of opties. For it may still be possible that this velocity of the missing wave is so snall, that the quantities on which it depends may be set equal to zero in the equations which represent the phenomena of optics. But the mental processes by which we satisfy ourselves of the validity of our results (if we do not work out the whole problem in the general case of no assumption in regard to the velocity of the missing wave) certainly involve conceptions of a higher degree of difficulty on account of the circumstances meniioned. Perhaps this ought not to affect our judgment with respect to the question of the tratb of the hypothesis.

Although the two theories give laws of exactly the same form for monochromatic light in the limiting case, their devia- 
tions from this limit are in opposite directions ; so that if the phenomena of optics differed in any marked degree from what we would have in the limiting case, it would be easy to find an experimentum erucis to decide between the two theories. A little consideration will make it evident that, when the principal indices of refraction of a crystal are given, the intermediate values for oblique wave-planes will be less if the velocity of the missing wave is small but finite than if it is infinitesimal, and will be greater if the velocity of the missing wave is very great but finite than if it is infinite*. Hence, if the velocity of the missing wave is small but finite, the intermediate values of the indices of refraction will be less than are given by Fresnel's law ; but if the velocity of the missing wave is very great but finite, the intermediate values of the indices of refraction will be greater than are given by Fresnel's law. But the recent experiments of Professor Hastings on the law of double refraction in Iceland spar do not encourage us to look in this direction for the decision of the question $\dagger$.

In a simple train of waves in a transparent medium, the potential energy, on the elastic theory, may be divided into two parts ; of which one is due to that general deformation of the æther which is represented by the equations of wavemotion, and the other to those deformations which are caused by the interference of the ponderable particles with the wavemotion, and to such displacements of the ponderable matter as may be caused, in some cases at least, by the motion of the ather. If we write $h$ for the amplitude, $l$ for the wavelength, and $p$ for the period, these two parts of the statical energy (estimated per unit volume for a space including many wave-lengths) may be represented respectively by

$$
\frac{\pi^{2} \mathrm{~B} h^{2}}{l^{2}} \text { and } \frac{b h^{2}}{4} \text {. }
$$

The sum of these may be equated to the kinetic energy, giving an equation of the form

$$
\frac{\pi^{2} \mathrm{~B} h^{2}}{l^{2}}+\frac{b h^{2}}{4}=\frac{\pi^{2} \mathrm{~A}^{\prime} h^{2}}{p^{2}} . . . .
$$

* This may be more clear if we consider the stationary waves formed by two trains of waves moving in opposite directions. The case then comes under the following theorem:-

"If the system undergo such a change that the potential energy of a given configuration is diminished, while the kinetic energy of a given motion is unaltered, the periods of the free vibrations are all increased, and conversely." See Lord Rayleigh's 'Theory of Sound,' vol. i. p. 85.

$\dagger$ Amer. Journ. Sci. rol. xxxiii. p. 60 . 


\section{Prof. J. W. Gibbs's Comparison of the Electric Theory}

$B$ is an absolute constant (the rigidity of the rether, previously represented by the same letter), $\mathrm{A}^{\prime}$ and $b$ will be constant (for the same medium and the same direction of the wave-normal) except so far as the type of the motion changes ; $i$. e. except so far as the manner in which the motion of the æether distributes itself between the ponderable molecules, and the degree in which these take part in the motion, may undergo a change. When the period of vibration varies, the type of motion will vary more or less, and $\mathrm{A}^{\prime}$ and $b$ will vary more or less.

In a manner entirely analogous*, the kinetic energy, on the electrical theory, may be divided into two parts; of which one is due to those general fluxes which are represented by the equations of wave motions, and the other to those irregularities in the fluxes which are caused by the presence of the ponderable molecules, as well as to such motions of the ponderable particles themselves, as may sometimes occur. These parts of the kinetic energy may be represented respectively by

$$
\frac{\pi \mathrm{F} l^{2} h^{2}}{p^{2}} \text { and } \frac{\pi^{2} f h^{2}}{p^{2}}
$$

Their sum equated to the potential energy gives

$$
\frac{\pi \mathrm{F} l^{2} h^{2}}{p^{2}}+\frac{\pi^{2} f h^{2}}{p^{2}}=\frac{\mathrm{G} h h^{2}}{4} . \text {. . . . . }
$$

Here $\mathrm{F}$ is the constant of electrodynamic induction, which is unity if we use the electromagnetic system of units; $f$ and $G$ (like $\mathrm{A}^{\prime}$ and $b$ ) vary only so far as the type of motion varies.

We have the means of forming a very exact numerical estimate of the ratio of the two parts into which the statical energy is thus divided on the elastic theory, or the kinetic energy on the electric theory. The means for this estimate is afforded by the principle, that the period of a natural vibration is stationary when its type is infinitesimally altered by any constraint $\uparrow$. Let us consider a case of simple wavemotion, and suppose the period to be infinitesimally varied, the wave-length will also vary, and presumably to some extent the type of vibration. But, by the principle just stated, if the æother or the electricity could be constrained to vibrate in the original type, the variations of $l$ and $p$ would be the same as in the actual case. Therefore, in finding the differential equation between $l$ and $p$, we may treat $b$ and $\mathrm{A}^{\prime}$ in (24) and

* See Amer. Journ. Sci. vol. xxïi. p. 262.

† See Lord Rayleigh's 'Theory of Sound,' vol. i. p. 84. The application of the principle is most simple in the case of stationary waves. 
$f$ and $\mathrm{G}$ in (25) as constant, as well as B and F. These equations may be written

Differentiating, we get

$$
\begin{gathered}
4 \pi^{2} \mathrm{~B} \frac{p^{2}}{l^{2}}+b p^{2}=4 \pi^{2} \mathrm{~A}^{\prime}, \\
\pi \mathrm{F} \frac{l^{2}}{p^{2}}+\frac{\pi^{2} f}{p^{2}}=\frac{1}{4} \mathrm{G} .
\end{gathered}
$$

$$
\begin{aligned}
4 \pi^{2} \mathrm{~B} d \frac{p^{2}}{\bar{l}^{2}} & =-b d\left(p^{2}\right), \\
\pi \mathrm{F} d \frac{l^{2}}{p^{2}} & =-\pi^{2} f d\left(p^{-2}\right) ;
\end{aligned}
$$

or

$$
\begin{aligned}
4 \pi^{2} \mathrm{~B} \frac{p^{2}}{l^{2}} d \log \frac{p^{2}}{l^{2}} & =-b p^{2} d \log p^{2}, \\
\pi \mathrm{F} \frac{l^{2}}{p^{2}} d \log \frac{l^{2}}{p^{2}} & =-\frac{\pi^{2} f}{p^{2}} d \log p^{-2} .
\end{aligned}
$$

Hence, if we write $\mathrm{V}$ for the wave-velocity $(l / p), n$ for the index of refraction, and $\lambda$ for the wave-length in vacuo, we have for the ratio of the two parts into which we have divided the potential energy on the elastic theory,

$$
\frac{b h^{2}}{4} \div \frac{\pi^{2} \mathrm{~B} h^{2}}{l^{2}}=\frac{d \log \mathrm{V}}{d \log p}=-\frac{d \log n}{d \log \lambda} ; .
$$

and for the ratio of the two parts into which we have divided the kinetic energy on the electrical theory, '

$$
\pi^{2} \frac{f h^{2}}{p^{2}} \div \frac{\pi \mathrm{F} l^{2} h^{2}}{p^{2}}=\frac{d \log \mathrm{V}}{d \log p}=-\frac{d \log n}{d \log \lambda} . .
$$

It is interesting to see that these ratios have the same value. This value may be expressed in another form, which is suggestive of some important relations. If we write $U$ for what Lord Rayleigh has called the velocity of a group of waves*,

$$
\left.\begin{array}{l}
\frac{\mathrm{U}}{\mathrm{V}}=1-\frac{d \log \mathrm{V}}{d \log l}, \\
\frac{d \log \mathrm{V}}{d \log l}=\frac{\mathrm{V}-\mathrm{U}}{\mathrm{V}}, \\
\frac{d \log \mathrm{V}}{d \log p}=\frac{\mathrm{V}-\mathrm{U}}{\mathrm{U}} .
\end{array}\right\} . . \cdot
$$

* See his "Note on Progressive Waves," Proc. Lond. Math. Soc. vol. ix. No. 125, reprinted in his 'Theory of 'Sound' vol. ii. p. 297. 
It appears, therefore, that in the elastic theory that part of the potential energy which depends on the deformation expressed by the equations of wave-motion bears to the whole potential energy the same ratio which the velocity of a group of waves bears to the wave-velocity. In the electrical theory, that part of the kinetic energy which depends on the motions expressed by the equations of wave-motion bears to the whole kinetic energy the same ratio.

Returning to the consideration of equations (26) and (27), we observe that in transparent bodies the last member of these equations represents a quantity which is small compared with unity, at least in the visible spectrum, and diminishes rapidly as the wave-length increases. This is just what we should expect of the first member of equation (27). But when we pass to equation (26), which relates to the elastic theory, the case is entirely different. The fact that the kinetic energy is affected by the presence of the ponderable matter, and affected differently in different directions, shows that the motion of the xther is considerably modified. This implies a distortion superposed upon the distortion represented by the equations of wave-motion, and very much greater, since the body is very fine-grained as measured by a wave-length. With any other law of elasticity we should suppose that the energy of this superposed distortion would enormously exceed that of the regular distortion represented by the equations of wavemotion. But it is the peculiarity of this new law of elasticity that there is one kind of distortion of which the energy is very small, and which is therefore peculiarly likely to occur. Now if we can suppose the distortion caused by the ponderable molecules to be almost entirely of this kind, we may be able to account for the smallness of its energy. We should still expect the first member of (26) to increase with the wavelength on account of the factor $l^{2}$, instead of diminishing, as the last member of the equation shows that it does. We are obliged to suppose that $b$, and therefore the type of the vibrations, varies very rapidly with the wave-length, even in those cases which appear farthest removed from anything like selective absorption.

The electrical theory furnishes a relation between the refractive power of a body and its specific dielectric capacity, which is commonly expressed by saying that the latter is equal to the square of the index of refraction for waves of infinite length. No objection can be made to this statement; but the great uncertainty in determining the index for waves of infinite length by extrapolation prevents it from furnishing any very rigorous test of the theory. Yet as the results of extra- 
polation in some cases agree strikingly with the specific dielectric capacity, although in other cases they are quite different, the correspondence is generally regarded as corroborative, in some degree, of the theory. But the relation between refractive power and dielectric capacity may be expressed in a form which will furnish a more rigorous test, as not involving extrapolation.

We have seen on page 249 how we may determine numerically the ratio of the two first terms of equation (25). We thus easily get the ratio of the first and last term, which gives

$$
\frac{\mathrm{G} h^{2}}{4}=\frac{d \log l}{d \log \lambda} \frac{\pi \mathrm{F} l^{2} h^{2}}{p^{2}} . . . . .
$$

In the corresponding equation for a train of waves of the same amplitude and period in vacuo, $l$ becomes $\lambda$, F remains the same, and for $G$ we may write $G^{\prime}$. 'This gives

Dividing, we get

$$
\frac{\mathrm{G} h^{2}}{4}=\frac{\pi \mathrm{F} \lambda^{2} h^{2}}{p^{2}} \text {. . . . . . . . }
$$

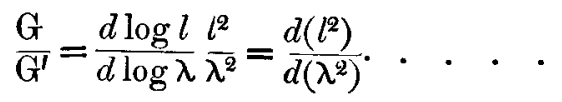

Now $G^{\prime}$ is the dielectric elasticity of pure æther. If $K$ is the specific dielectric capacity of the body which we are considering, $G^{\prime} / K$ is the dielectric elasticity of the body and $G^{\prime} / 2 K$ is the potential energy of the body (per unit of volume), due to a unit of ordinary electrostatic displacement. But $\mathrm{G} h h^{2} / 4$ is the potential energy in a train of waves of amplitude $h$. Since the average square of the displacement is $h^{2} / 2$, the potential energy of a unit displacement such as occurs in a train of waves is G/2. Now in the electrostatic experiment the displacement distributes itself among the molecules so as to make the energy a minimum. But in the case of light the distribution of the displacement is not determined entirely by statical considerations. Hence

$$
\left.\begin{array}{l}
\frac{G}{2} \geqq \frac{G^{\prime}}{2 K^{\prime}}, \\
K \geqq \frac{G^{\prime}}{G},
\end{array}\right\} \quad \cdot \quad \cdot . \cdot . \cdot
$$

and

$$
\mathrm{K} \geqq \frac{d\left(\lambda^{2}\right)}{d\left(l^{2}\right)} \text {. } \quad \cdot \quad \cdot \quad \cdot \quad \cdot \text {. }
$$

It is to be observed that if we should assume for a dispersionformula

$$
n^{-2}=a-b \lambda^{-2}, \text {. . . . . }
$$


$1 / a$, which is the square of the index of refraction for an infinite wave-length, would be identical with the second member of (33).

Another similarity between the electrical and optical properties of bodies consists in the relation between conductivity and opacity. Bodies in which electrical fluxes are attended with absorption of energy absorb likewise the energy of the motions which constitute light. This is strikingly true of the metals. But the analogy does not stop here. To fix our ideas, let us consider the case of an isotropic body and circularly polarized light, which is geometrically the simplest case, although its analytical expression is not so simple as that of plane-polarized light. The displacement at any point may be symbolized by the rotation of a point in a circle. The external force necessary to maintain the displacement $\mathfrak{F}$ is represented by $n^{-2} \mathfrak{F}$. In transparent bodies, for which $n^{-2}$ is a positive number, the force is radial and in the direction of the displacement, being principally employed in counterbalancing the dielectric elasticity, which tends to diminish the displacement. In a conductor $n^{-2}$ becomes complex, which indicates a component of the force in the direction of $\dot{\mathfrak{F}}$, that is, tangential to the circle. This is only the analytical expression of the fact above mentioned. But there is another optical peculiarity of metals, which has caused much remark, viz. that the real part of $n^{2}$ (and therefore of $n^{-2}$ ) is negative, $i$. e. the radial component of the force is directed towards the centre. This inwardly directed force, which evidently opposes the electrodynamic induction of the irregular part of the motion, is small compared with the outward force which is found in transparent bodies, but increases rapidly as the period diminishes. We may say, therefore, that metals exhibit a second optical peculiarity-that the dielectrical elasticity is not prominent as in transparent bodies. This is like the electrical behaviour of the metals, in which we do not observe any elastic resistance to the motion of electricity. We see, therefore, that the complex indices of metals, both in the real and imaginary part of their inverse squares, exhibit properties corresponding to the electrical behaviour of the metals.

The case is quite different in the elastic theory. Here the force from outside necessary to maintain in any element of volume the displacement $\left(\mathcal{F}\right.$ is represented by $n^{2} \ddot{\mathcal{E}}$. In transparent bodies, therefore, it is directed toward the centre. In metals, there is a component in the direction of the motion $\dot{\mathfrak{F}}$, while the radial part of the foree changes its direction and is often many times greater than the opposite force in transparent bodies. This indicates that in metals the displacement of 
the æther is resisted by a strong elastic force, quite enormous compared to any thing of the kind in transparent bodies, where it indeed exists, but it is so small that it has been neglected by most writers, except when treating of dispersion. We can make these suppositions, but they do not correspond to anything which we know independently of optical experiment.

It is evident that the electrical theory of light has a serious rival, in a sense in which, perhaps, one did not exist before the publication of Sir William Thomson's paper in November last*. Nevertheless, neither surprise at the results which have been achieved, nor admiration for that happy audacity of genius, which, seeking the solution of the problem precisely where no one else would have ventured to look for it, has turned half a century of defeat into victory, should blind us to the actual state of the question.

It may still be said for the electrical theory, that it is not obliged to invent hypotheses $t$, but only to apply the laws furnished by the science of electricity, and that it is difficult to account for the coincidences between the electrical and optical properties of media, unless we regard the motions of light as electrical. But if the electrical character of light is conceded, the optical problem is very different from anything which existed in the time of Fresnel, Cauchy, and Green. The third wave, for example, is no longer something to be gotten rid of quocunque modo, but something which we must dispose of in accordance with the laws of electricity. This would seem to rule out the possibility of a relatively small velocity for the third wave.

* "Since the first publication of Cauchy"s work on the subject in 1830, and of Green's in 1837, many attempts have been made by many workers to find a dynamical foundation for Fresnel's laws of reflexion and refraction of light, but all hitherto ineffectually." Sir William Thomson, loc. cit.

"So far as I am aware, the electric theory of Maxwell is the only one satisfying these conditions (of explaining at once Fresnel's laws of double refraction in crystals and those governing the intensity of reflexion when light passes from one isotropic medium to another)." Lord Rayleigh, Phil. Mag. September 1888.

$\dagger$ Electrical motions in air, since the recent experiments of Professor Hertz, seem to be no longer a matter of hypothesis. We can hardly suppose that the case is essentially different with the so-called vacuum. The theorem that the electrical motions of light are solenoidal, although it is convenient to assume it as a hypothesis and show that the results agree with experiment, need not occupy any such fundamental position in the theory. It is in fact only another way of saying that two of the constants of electrical science have a certain ratio (infinity). It would be easy to commence without assuming this value, and to show in the course of the development of the subject that experiment requires it, not of course as an abstract proposition, but in the sense in which experiment can be said to require any values of any constants, that is, to a certain degree of approximation. 